ORIENTAL JOURNAL OF
ISSN: 0974-6471
March 2017,
Col. 10, No. (1):
Pgs. 227-231

\title{
A Comparative Review on Different Methods of Face Recognition
}

\author{
TENZIN DAWA ${ }^{1}$ and N.VIJAYALAKSHMI ${ }^{2 *}$ \\ 'Department of Computer Science, Master of Computer Application, Christ University, India. \\ ${ }^{2}$ Department of Computer Science, Faculty of Computer Science, Christ University, India. \\ ${ }^{*}$ Corresponding author E-mail: vijayalakshmi.nair@christuniversity.in
}

http://dx.doi.org/10.13005/ojcst/10.01.31

(Received: March 16, 2017; Accepted: March 17, 2017)

\begin{abstract}
Face Recognition is a biometric system which can be used to identify or verify a person from digital image by using the facial features that are unique to each other. There are many techniques which can be used in a face recognition system. In this paper we review some of the algorithms and compare them to see which technique is better compared to one another. Techniques that are compared in this technique are Non-negative matrix factorization (NMF) with Support Vector Machine (SVM), Partial Least Squares (PLS) with Hidden Markov Model (HMM) and Local Ternary Pattern (LTP) with Booth's Algorithm.
\end{abstract}

Keywords: Face Recognition, NMF, SVM, PLS, HMM, LTP, Booth Algorithm.

\section{INTRODUCTION}

Security is a major concern in day to day life. Biometrics systems are providing an aid to solve this problem. Among the various biometrics available, face recognition is a wellaccepted biometric software application capable of identifying faces and verifying faces from a given set of database. Face recognition can be used from anything like a frame from a video or a digital image. Face recognition is mostly used for security systems and commercial identification and is considered to be better than other biometrics such as iris recognition and fingerprint. Features of face are extracted and implemented through algorithms which are processed and are compared in the database, if the face exist or a similar face is in the database then the system can display the image or else it is unknown or not existing in the database. There are various approaches to increase the recognition rate of faces. This paper does a comparative study on various approaches of face recognition.

\section{Applications of face recognition Access Control}

Verifying a face, matching it against a single enrolled face in the database is well within the capabilities of laptops and PCs being used now a days which helps in ease of use of this 
biometric over password protection. Since most of available biometric systems are of third party, systems does not have hardware password as well as hardware security and full access afforded by boot-time. Banks have been very conservative in deploying biometrics as they don't want to risk losing customers in being falsely rejected on a critical time for that customer or the risk of falsely identifying someone pretending to be a customer, and customer themselves are reluctant to indulge in the additional security process as well.

\section{Identification Systems}

In some states in USA they are testing face recognition for welfare benefits where they compare the entire database when enrolling a new applicant in welfare to make sure that the applicant only has one identity. Unfortunately our current face recognition systems are not reliable enough to identify a single person from millions of people enrolled in the, so currently other information of the person such as name, age etc are taken into consideration to narrow the search pool, which means still human intervention is required to review the results produced by the system to prevent false alarm.

\section{Surveillance}

Surveillance domain is probably the place which is generating more interest than any other domain, and because of the type of the information that is in a video format, video is becoming the medium of choice for surveillance.so for any form of application that requires identification face recognition is the best biometric system.

In this paper we are going to review algorithms that are used for face recognitions: Nonnegative Matrix Factorization (NMF) with Support Vector Machine (SVM), Partial Least Squares (PLS) with Hidden Markov Model (HMM) and Local Ternary Pattern with Booth's Algorithm.

\section{Steps Involved in Face Recognition Face Detection}

In face detection, algorithms tend to focus more on front of the human face for detection, where as in image detection the image of a person is matched bit by bit and will be stored in the database but the matching process will become invalid with any changes. When an input image is given face detection algorithm is responsible to check if any face is there or not and where that human face is located in the given input.

\section{Feature Extraction}

The process involved in feature extraction is to reduce the amount of data required to describe a large set of data while not losing out the important aspects of the data. On a complex set of data majority of problem arises from number of variables which require more memory and more computational power while it might also make the classification algorithm to over fit to the sample set for training and perform poorly when new set of data is given.Feature extraction is a general term where combinations of variable are constructed to get around the problems, extracting a distinctive attribute or aspect of something in form of data.

\section{Face Recognition}

After extracting the feature of each face through feature extraction, the last step is to recognize the identities of these faces from the database which is required. In this database several images for each person is stored as well as the features extracted from that image, so when a input image comes in, face detection and feature extraction are performed on it and the $n$ they are compared to the images stored in the database.

In general there are two applications of face recognition, first is identification and the second one is verification. Face identification is mostly used by corporate who wants the system to tell if he/she

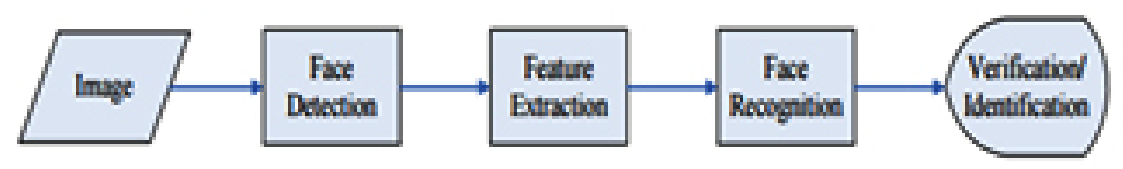

Fig. 1: Configuration of general face recognition structure. ${ }^{1}$ 
is working here or not while in face verification on a given image the system should give true or false indication on the guess of identification the person made.

\section{literature review}

Lian and $\mathrm{Er}^{2}$ in their paper, referred to several latest approaches of representing each variation of faces in detail and also comparing all these approaches as well, a detailed study on LBP is done and to check how face recognition works under various illumination, various combination with other techniques are also done. It was noted in their paper that when there is only one image per person then most of the techniques does not give satisfactory performance compared with when it is done with more than one image per person, so when LPB is combined with other techniques it makes itbetter way to deal with illumination variances.

Qingzhou Zhang, Ziqiang Wang and Xia $S^{3}{ }^{3}$ in their paper they proposed an efficient face recognition algorithm by using Non-negative Matrix Factorization (NMF) and SupportVector Machine (SVM). First the face subspace was obtained by using dimension reduction algorithm NMF after which new face is kept on that face subspace for recognition purpose then SVM classifier is applied on the image to classify the new face image. SVM is usually applied to find optimal line separating hyperplane so that the closest vectors of both classes is maximized and in order to make SVM applicable to nonlinear separable face data, the kernel-based methods is used through kernel function because kernel function can be applied without knowing the nonlinear mapping explicitly and experimental results are also pointing out the result of their proposed method of algorithm.

In the paper proposed by Benyong Liu and Yegang $\mathrm{Hu}^{4}$ they presented a scheme which includes three main elements: First one is preprocessing based on pixel averaging for dimension reduction and then apply energy normalization to minimize the effect of image brightness, feature extraction can be successfully conducted by methods like Fast Fourier Transform and PCA or with Partial Least Square, and then finally the classification is done with Hidden Markov Model. Pixel averaging is a method of substituting neighboring pixel by a single pixel which is acquired by finding the mean of the neighbor pixels and then energy normalization is adopted for a down-sampled image to reducethe brightness effect of the given image.

In the paper proposed by Abdullah Gabbi, Mohammad Fazle Azeem and Nishatbanu Z H Nayakwadi $^{5}$, they proposed a particular process which makes use of Local ternary pattern and Booth's Algorithm techniques to detect or to record the local face features, which take advantage of central pixel for computation of the feature. The face of the images are separatedinto small windows and since classification can be employed better with local descriptors, a Non- overlapping block wise processing is done on image to limit the features. The Support Vector Machine (SVM) and KNN classifier with proposed similarity measure is used for face recognition. Finally, ROC and CMC are plotted for analysis of the system.

The experiment that was done on face 94 and ORL dataset states that the method proposed by them has a higher accuracy rate on classification than most of the previously proposed methods.

Quanbin Li, Chaunwie Sun and JingaoLiu' ${ }^{6}$, in their proposed application, face texture features are obtained with the help of cascading of two Local Binary Pattern features. Then, face recognition under the complex light is achieved by the use of SVM classifier. First, the local detail texture features of the sub-block faces are secured by using relatively small LBP operators. Then after that, global profile characteristics are obtained by the use of relatively large LBP operators. In the end, two histograms which is obtained are combined into one in the correct order as the final identification feature.

Swati Y.Raut, Dipti.A.Doshi ${ }^{7}$, they proposed a novel algorithm based on E-HMM and discriminating set, their algorithm is divided in two sections, the first one is a training module and the second is the assembly module, and their paper has a very high recognition rate compared to other methods. 


\section{Algorithms Used in this Paper \\ Non-negative matrix factorization (NMF)}

In non-negative matrix factorization the returned value will be a non-negative value and with the underlying constrains which are non-negative, it is able to lean on the localized parts based representation while non-negativity part makes the output matrices easier to inspect and the solution is approximated numerically since the given problem is not exactly solvable.

\section{Support Vector Machine (SVM)}

SVM is one of the supervised machine learning algorithm which can be used in two application i.e. regression challenges or for classifications but is mostly used for classification purposes. This algorithm assumes that the given data is linearly separable and it attempts to find an optimal vector (a line) which will differentiate the two classes.

\section{Partial Least Square (PLS)}

PLS is an extension of multiple linear regression which is used to find the fundamental relations between variable in the form of matrix, a latent variable is chosen in the $X$ space such that it provides maximum correlation with $Y$ space, the dependent variable. PLS is also one the least restrictive compared with other extensions that are available with multiple linear regression model and because of the flexibility it can be more accurate than other traditional multivariate methods for example when there are less number of observations that that of predictor variables..

\section{Hidden Markov Model (HMM)}

A hidden Markov model (HMM) is heavily based on statistical approach, one in which sequence of date is in unobserved state and the states through which the model went to generate that unobserved state is also not known ${ }^{9}$. The sequence of the unobserved state is approximated numerically in the hidden Markov model through the already available observed state.
Table 1: Comparisons of Algorithms

The comparative study of face recognition that are discussed in this paper

\begin{tabular}{lcc}
\hline Algorithm & Database Used & Result \\
\hline NMF with SVM & Yale & $79.10 \%$ (avg) \\
NMF with SVM & ORL & $94.33 \%$ (avg) \\
PLS with HMM & ORL & $80.83 \%$ (avg) \\
LTP with Booth & ORL & $71.78 \%$ (avg) \\
LTP with Booth & Faces94 & $73.37 \%$ (avg) \\
\hline
\end{tabular}

\section{Local Ternary Pattern}

LTP is an extension of another algorithm known as Local binary patterns (LBP). LTP helps in overcoming limitations of local binary pattern such as sensitivity to random and quantization noise in uniform image regions like cheeks and forehead, it also retains the advantages of Local binary pattern as well. In this algorithm it thresholds pixels into three values instead of two as in local binary pattern, the neighboring pixels are combined after thresholding into a ternary pattern thus encodes the small pixel difference into separate state ${ }^{10}$.

\section{Booth's Algorithm}

Booth's algorithm mainly servers two purposes i.e., fast multiplication and signed multiplication. Fast multiplication happens when there are two or more 0's or 1's in consecutive order in the multiplier, Booth's multiplication algorithm multiplies two signed numbers in two's compliment notation.

\section{CONCLUSION}

This paper presented an independent, comparative study of six face recognition algorithm combined into three methods. Face recognition is an area where people are showing interest are growing and this paper provides a way which can be understand by all the users in a simple and informative way on face recognition. The NMF with SVM yields $94.33 \%$ on ORL database and is the best among the algorithms compared here followed by PLS with HMM on the same database. 


\section{REFERENCES}

1. We i-Lun Chao, "Face Recognition",GICE, National Taiwan University.

2. LianZhichao and ErMengJoo, "Face Recognition Under Varying Illumination", NanyangTechnilogical University, Singapore.

3. Xia Sun, Qingzhou Zhang and Ziqiang Wang"Face Recognition Based on NMF and SVM", Wuhan University of Technology and Henan University of Technology, China, 2009.

4. Yegang $\mathrm{Hu}$, Benyong Liu "Face Recognition Based on PLS and HMM", Guizhou University, China,2009.

5. Abdullah Gubbi, Mohammad FazleAzeem and Nishabanu Z H Nayakwadi "Face recognition using Local Ternary Pattern and Booth's Algorithm", $3^{\text {rd }}$ International Conference on Eco-Friendly Computing and Communication Systems, 2014.
6. Quanbin Li, ChuanweiSun,Jingao Liu "Illumination Invariant Face Recognition Based on ULBP and SVM", JSNU, ECNU, China $5^{\text {th }}$ International Conference on BioMedical Engineering and Informatics (BMEl 2012).

7. Swati Y.Raut, Dipti.A.Doshi "A Face Recognition System by Hidden Markov Model and Discriminating Set Approach"

8. MenakaRajapakse and Lonce Wyse "Face Recognition with Non-negative Matrix Factorization",Institute for Infocomm Research, Singapore.

9. https://in.mathworks.com/help/stats/hiddenmarkov-models-hmm.html

10. K. Srinivasa Reddy, V.Vijaya Kumar, B. Eswara Reddy "Face Recognition Based on Texture Features using Local Ternary Patterns", Hyderabad, Hyderabad, A.P.,India.,2015 